This document is confidential and is proprietary to the American Chemical Society and its authors. Do not copy or disclose without written permission. If you have received this item in error, notify the sender and delete all copies.

\title{
Substrate Topography Modulates Cell Aging on a Progeria Cell Model
}

\begin{tabular}{|c|c|}
\hline Journal: & ACS Biomaterials Science \& Engineering \\
\hline Manuscript ID & $a b-2018-002244 . R 1$ \\
\hline Manuscript Type: & Letter \\
\hline Date Submitted by the Author: & $\mathrm{n} / \mathrm{a}$ \\
\hline Complete List of Authors: & $\begin{array}{l}\text { Pitrez, Patricia; Center for Neuroscience and Cell Biology; Institute for } \\
\text { Biomedical Imaging and Life Sciences (IBILI), Faculty of Medicine } \\
\text { Estronca, Luís; Center for Neuroscience and Cell Biology; Institute for } \\
\text { Biomedical Imaging and Life Sciences (IBILI), Faculty of Medicine } \\
\text { Vazão, Helena; Center for Neuroscience and Cell Biology } \\
\text { Egesipe, Anna-Laure; CECS, I-STEM, AFM, Institute for Stem Cell Therapy } \\
\text { and Exploration of Monogenic diseases } \\
\text { Le Corf, Amélie; CECS, I-STEM, AFM, Institute for Stem Cell Therapy and } \\
\text { Exploration of Monogenic diseases } \\
\text { Navarro, Claire; Aix Marseille Univ, INSERM, GMGF } \\
\text { Levy, Nicolas; Aix Marseille Univ, INSERM, GMGF; Molecular Genetics } \\
\text { Laboratory, Department of Medical Genetics, La Timone Children's hospital } \\
\text { De Sandre-Giovannoli, Annachiara; Aix Marseille Univ, INSERM, GMGF; } \\
\text { Molecular Genetics Laboratory, Department of Medical Genetics, La Timone } \\
\text { Children's hospital } \\
\text { Nissan, Xavier; CECS, I-STEM, AFM, Institute for Stem Cell Therapy and } \\
\text { Exploration of Monogenic diseases } \\
\text { Ferreira, Lino; Center for Neuroscience and Cell Biology; Institute for } \\
\text { Biomedical Imaging and Life Sciences (IBILI), Faculty of Medicine }\end{array}$ \\
\hline
\end{tabular}

\section{SCHOLARONE ${ }^{\text {th }}$}

Manuscripts 


\title{
Substrate Topography Modulates Cell Aging on a
}

\section{Progeria Cell Model}

\author{
Patricia R. Pitrez ${ }^{1,2 \ddagger}$, Luís Estronca ${ }^{1,2 \ddagger}$, Helena Vazão ${ }^{1}$, Anna-Laure Egesipe ${ }^{3}$, Amélie Le Corf ${ }^{3}$, \\ Claire Navarro ${ }^{4}$, Nicolas Lévy ${ }^{4,5}$, Annachiara De Sandre-Giovannoli ${ }^{4,5}$, Xavier Nissan ${ }^{3}$, Lino \\ Ferreira $^{1,2 *}$ \\ ${ }^{1}$ Center for Neuroscience and Cell Biology, University of Coimbra, Coimbra, Portugal \\ ${ }^{2}$ Institute for Biomedical Imaging and Life Sciences (IBILI), Faculty of Medicine, University of \\ Coimbra, Coimbra, Portugal \\ ${ }^{3}$ CECS, I-STEM, AFM, Institute for Stem Cell Therapy and Exploration of Monogenic diseases, \\ Evry cedex, France \\ ${ }^{4}$ Aix Marseille Univ, INSERM, GMGF, Marseille, France \\ ${ }^{5}$ Molecular Genetics Laboratory, Department of Medical Genetics, La Timone Children's \\ hospital, Marseille, France.
}

KEYWORDS: aging, topography, extracellular matrix, progeria, smooth muscle cells

Aging is characterized by a progressive accumulation of cellular damage, which leads to impaired function. Little is known whether substrates can influence cell aging. This is of utmost importance in the development of medical devices that are in contact with human tissue for long periods of time. To address this question, we have used an accelerated aging cell model derived from Hutchinson-Gilford Progeria Syndrome (HGPS) induced pluripotent stem cells (iPSCs). Our results show that HGPS-iPSC smooth muscle cells (SMCs) have an increased aging profile in substrates with specific micropatterns than in flat ones. This is characterized by an up- 
regulation in the expression of progerin, $\beta$-galactosidase, annexin 3 and 5 and caspase 9 . Signs of cell aging are also observed in SMCs without HGPS cultured in substrates with specific microtopographies. It is further showed that specific micropatterned substrates induce cell aging by triggering a DNA damage program likely by the disruption between cyto- and nucleoskeleton.

The control of cell-medical device interactions at the bio-interface is important to modulate many aspects in cell biology including cell adhesion and cell function ${ }^{1-3}$. An aspect that is less studied is whether the topography of the medical devices such as stents, vascular grafts/patches, catheters, among others, modulates cell aging. Cell aging is characterized by several hallmarks including genomic instability, telomere attrition, epigenetic alterations, loss of proteostasis, deregulated nutrient sensing, mitochondrial dysfunction, cellular senescence, stem cell exhaustion and altered intercellular communication ${ }^{4}$. A better understanding of substrate topography effect in cell aging may lead to the development of enhanced medical devices and material-tissue interfaces.

To evaluate the effect of topography in cell aging we have used an accelerated aging cell model derived from Hutchinson-Gilford Progeria Syndrome (HGPS) to reduce the time of the experiments. HGPS is a rare and fatal genetic disease with an accelerated aging process ${ }^{5-7}$. This disease is caused by a single point mutation of the LMNA gene that encodes the major components of the nuclear lamina, the lamins $\mathrm{A}$ and $\mathrm{C}^{7}$. This mutation leads to the production and accumulation of an abnormally truncated lamin A protein called progerin. Because of progerin accumulation, HGPS cells present nuclear blebs, disorganization and telomere shortening which leads to DNA damage, genomic instability and premature senescence ${ }^{6}$. 
Importantly, it has been shown that progerin also accumulates in normally aged individuals ${ }^{8}$ and is associated with vascular aging ${ }^{9}$. Dysfunction of SMCs appears to occur as age progresses ${ }^{10}$. Aged SMCs have altered proliferative and apoptotic behavior and response to growth factors ${ }^{7}$. In addition, in pathologic aging (HGPS patients), SMCs are partially lost in large arterial vessels causing cardiovascular disease and premature death ${ }^{11}$. In the setting of this work, we have used SMCs derived from HGPS-iPSCs and non-disease iPSCs (N-iPSCs) ${ }^{5}$ as a cell model due to our expertise in the derivation of SMCs from human pluripotent stem cells ${ }^{12,13}$, our interest in vascular aging ${ }^{14}$, response of SMCs to mechanical stress ${ }^{11}$ and the central role of these cells in restenosis after the use of medical stents ${ }^{15}$. Our hypothesis is that ECM topography may disturb the biomechanical response of the cell and thus accelerate cell aging. This effect might depend on the "aging status" of the cell and on whether cells aged by a physiologic or pathological process.

Although several substrates with topographic elements have been used to study the effect on SMCs differentiation, SMCs alignment and nuclear shape ${ }^{16-18}$, no study has evaluated the effect of topography in the aging of SMCs. Here, we have studied the aging process of SMCs with and without HGPS in micropatterned substrates with different geometries (Figure 1A). Poly(dimethylsiloxane) (PDMS) was chosen as substrate because it is present on the surface of balloon catheters, guiding catheters, hemostatic valves, and also stents ${ }^{19}$, it is a translucent elastomer with optical characteristics needed for microscopy and it is relatively non-cytotoxic. We have chosen micropatterned PDMS substrates having grooves/ridges with sub-cellular dimensions (four different widths: 2, 3, 4 and $5 \mu \mathrm{m}$; with one height: $1.5 \mu \mathrm{m}$ ) (Figure 1A) because cells respond to these substrates by altering their morphology and thus facilitating the evaluation of the topography in cell biology. Since the diameter of SMCs is around $20 \mu \mathrm{m}^{11}$, we 
generated substrates with a groove width between 2 and $5 \mu \mathrm{m}$ while keeping constant the height $(1.5 \mu \mathrm{m})$ (Figure S1A). An height of $1.5 \mu \mathrm{m}$ was chosen because it was demonstrated previously to be effective to enhance cell alignment on parallel lines ${ }^{20}$. Substrates with groove's width of 1 $\mu \mathrm{m}$ were not tested because they were in the range of the resolution of the lithography technique used and thus the fidelity of the micropatterning was not warranted. Groove widths above $5 \mu \mathrm{m}$ were not tested because previous studies have shown much less SMCs alignment in those conditions $^{18}$. Each micropatterned PDMS was labeled considering the width of the groove and the width of the ridges $\left(2 \_2\right.$ means $2 \mu \mathrm{m}$ for the width of the groove and $2 \mu \mathrm{m}$ for the width of the ridge). PDMS without micropatterns (flat) was used as control.

HGPS-iPSCs and N-iPSCs were differentiated into SMCs using a three-step protocol (Figure S1B). At this stage, more than $95 \%$ of both differentiated cells express $\alpha$-smooth muscle actin ( $\alpha$-SMA), smooth muscle myosin heavy chain (SMMHC) and calponin proteins, markers of SMCs ${ }^{13}$. Moreover, HGPS-iPSC SMCs express progerin protein $(30 \%$ of the cells). These cells were then cultured in micropatterned PDMS substrates or PDMS without micropatterns (flat). Cell adhesion was quantified after contact of the cells with the substrates for $6 \mathrm{~h}$ (Figure S1C). Both cell types adhered to the substrates. No statistically significant differences were found in cell adhesion on flat or micropatterned substrates. Cells showed similar number of focal adhesions on flat and micropatterned substrates (Figure S1D).

We then asked whether substrate topography could affect the organization of cellular contractile machinery. Studies have shown that SMCs cultured in micropatterned substrates have a higher contractile phenotype, as confirmed by the expression and organization of contractile proteins ( $\alpha$-SMA, SMMHC and calponin), than unpatterned cells ${ }^{17,21,22}$. In this study we 
evaluated the expression of calponin in HGPS-iPSC SMCs and N-iPSC-SMCs cultured in micropatterned and flat substrates for 15 days. We have selected 15 days as a compromise between time to have a biological effect and enough number of cells for characterization due to the rapid aging process in HGPS-iPSC SMCs. Because more than $90 \%$ of the cells expressed calponin before culture on micropatterned substrates (although only 10\% of the cells had individual organized calponin fibers) we evaluated the impact of the substrate in the organization of calponin fibers (Figure S2). In general (3 out of 4 substrates), both cell types showed a higher number of organized calponin fibers in micropatterned than in flat substrates and thus a higher contractile phenotype. It is interesting to note that the topographies that induce higher number of calponin fibers in HGPS-iPSC SMCs (2_2; 4_4 and 5_5) are not the same as in N-iPSC-SMCs (2_2; $3 \_3$ and 4_4). Altogether, substrate topography induced a contractile phenotype in both HGPS-iPSC SMCs and N-iPSC SMCs.

Cell nucleus interacts with the cytoskeleton and deforms according to the stresses from the $\mathrm{ECM}^{22}$. To evaluate the effect of topography on SMCs alignment, HGPS-iPSC SMCs and NiPSC SMCs were cultured in flat and micropatterned substrates for 15 days and nuclei alignment was quantified (Figure 1B). Both cells show no preferred alignment on flat substrates (Figure 1C.1 and 1C.2). In contrast, both cell types aligned their nuclei along the major axis of the micropatterned substrate (Figure 1C.1 and 1C.2). The percentage of both cells showing an alignment angle of less the $10^{\circ}$ relative to the major axis of the micropatterned was quantified (Figure 1C.3). With the exception of substrate 3_3, HGPS-iPSC SMCs showed lower levels of nuclei alignment than N-iPSC SMCs, likely due to an increase in stiffening of HGPS nucleoskeleton and less flexibility to deform along the microstructures ${ }^{23}$. Nevertheless, the 
degree of cell nuclei alignment on micropattern samples was significantly higher than the degree of nuclei alignment on flat conditions.

Cells translate mechanical stimuli or spatial alignment into biochemical signals through a complex mechanosensing system coupling focal adhesions, cytoskeleton and the nucleus (Figure 2A). In this system, lamins interact with a variety of nuclear envelope proteins including emerin, nesprin and SUN families, as well as several transcriptional regulators ${ }^{24}$. All these molecular players are components of the LINC complex that connects the nuclear lamina and envelope with the cytoskeleton ${ }^{25}$. Disruption of this complex can result in impaired force transmission between the cytoskeleton and the nuclei ${ }^{26}$. Therefore, we evaluated the connection between cytoskeleton and nuclei by two different approaches ${ }^{26}$. In the first approach we calculated both the angle between the long axis of the cell nuclei and the long axis of the cytoplasm to the micropattern followed by the ratio of cells having an angle below $10^{\circ}$ (considered aligned to the micropattern; please see Supporting Information) (Figure 2B). Our results show that HGPS-iPSC SMCs cultured in substrates 2_2, 3_3 and 5_5 (in particular 2_2 and 5_5) have a higher cytoplasm alignment than nuclei alignment to the micropattern, suggesting a decoupling between cytoskeleton and nucleoskeleton. In the second approach, we measured nuclear deformation in response to the micropatterns (Figure 2C). Previously, it was shown that the disruption of LINC complex reduces nuclear deformation in response to extracellular strain application ${ }^{26}$. Our results show that HGPS-iPSC SMCs have a lower nuclear elongation than N-iPSC SMCs in 3 out of 4 substrates, and thus suggesting a higher disruption of LINC complex. Taken together, using two indirect approaches, our results suggest that the LINC complex might be impaired in HGPSiPSC SMCs cultured in micropatterned substrates. These results are in line with recent studies showing that HGPS fibroblasts cultured in micropatterned substrates have an impairment in 
LINC complex ${ }^{27}$. Our results do not exclude the possibility that differences in cell and nuclear size as well as nuclear stiffness between HGPS-iPSC SMCs and N-iPSC SMCs may account for some of the observed differences.

We then asked whether the expression of progerin and other aging markers modulate this mechanosensing mechanism. Therefore, we evaluated the expression of LINC complex genes in HGPS-iPSC SMCs cultured in flat and 5_5 micropatterned substrates (as a representative micropatterned substrate, see below). These markers included nesprin family (nesprin 1,2 and 3; SYNE1, SYNE2, SYNE3), SUN-domain proteins (SUN1 and SUN2), emerin (EMD) and lamin A $(L M N A)^{24}$ (Figure S3A). Cells cultured on micropatterned substrates or flat substrates had minor differences in the expression of Nesprin and SUN genes (Figure S3B). However, the expression and localization of SUN1 and Nesprin2 proteins was different between cells cultured on micropatterned substrates and flat substrates. Our results showed an increase in SUN1 at both nuclear and cytoplasm sites for cells cultured in micropatterned substrates relatively to flat substrates (Figure S3C). Our data corroborate previous results showing that HGPS cells expressing progerin accumulate SUN1 in the nucleus ${ }^{28,29}$. The increase in SUN1 protein was not due to elevated SUN1 mRNA levels (Figure S3B) but likely due to reduced protein turnover, as demonstrated before for HGPS-fibroblasts ${ }^{28}$. We also analyzed the distribution of Nesprin2 protein. Our results showed an accumulation of this protein in the nuclear envelope and cytoplasm of HGPS iPSC-SMCs cultured on micropatterned substrates relatively to flat substrates. The accumulation of SUN1 and Nesprin2 proteins may be a cell compensatory mechanism for the disruption of the LINC complex. Overall, our results show that progerin modulate the mechanosensing mechanism, specifically altering the expression and location of LINC complex proteins. 
We then asked whether the substrate topography could enhance cell aging. To evaluate cell aging, both HGPS-iPSC SMCs and N-iPSC SMCs were cultured in micropatterned and flat substrates for 15 days. Our results show that HGPS-iPSC SMCs have statistically significant higher expression of progerin protein than cells cultured in flat substrates (Figure 3A.1). For cells cultured in substrates $2 \_2$ and 5_5, the expression of progerin protein was twice of the expression (30\% versus ca. $60 \%$ ) observed in cells cultured in flat substrates (Figure 3A.2). The up-regulation of progerin in HGPS-iPSC SMCs cultured on micropatterned substrates was also confirmed at gene level (Figure 3B). We also evaluated the effect of the substrate topography in the aging of N-iPSC SMCs. In this case, the aging effect was relatively low (below 10\%) for all the conditions tested, and only cells cultured in substrates 5_5 showed a statistically significant increase in progerin expression (from 2.5\% to 7\%) (Figure 3A.3).

Next, we extended aging analyzes in HGPS-iPSC SMCs and N-iPSC-SMCs cultured in micropatterned and flat substrates to mRNA transcripts of $G L B 1$ (encoding $\beta$-galactosidase) ${ }^{30}$, ANXA3 (encoding annexin 3) ${ }^{31}, A N X A 5$ (encoding annexin 5) ${ }^{32}$ and CASP9 (encoding caspase $9)^{33}$, genes associated with cell aging. All these aging markers were upregulated when HGPSiPSC SMCs were cultured on micropatterned substrates 4_4 and 5_5 compared to flat substrates (Figure 3C). In most cases, the level of gene expression is similar to the one observed for human fibroblasts isolated from HGPS patients. These results show that HGPS-iPSC SMCs cultured on micropatterned structures presented a higher aged phenotype relatively to cells cultured in flat substrates. According to the expression of progerin and aging gene expression results, the substrate 5_5 was the substrate that yielded the most effective aging in HGPS-iPSC SMCs. These results were further confirmed by the evaluation of $\beta$-galactosidase at protein level and cell proliferation. Cells cultured in the substrate 5_5 had higher expression of $\beta$-galactosidase 
than flat substrate (Figure S4A) as well as lower proliferation (Figure S4B). Moreover, we evaluated the aging in N-iPSC SMCs cultured in flat or micropatterned (substrate 5_5) substrates (Figure S5). With the exception of $A N X A 3$ gene, N-iPSC SMCs cultured in substrate 5_5 showed relatively low expression for all the aging markers. Overall, our results show that HGPSiPSC SMCs cultured on micropatterned substrates, particularly in the 5_5 substrate, have increased accumulation of progerin, senescence markers including GLB1, ANXA3, ANXA5 and CASP9 mRNA transcripts and $\beta$-galactosidase protein than HGPS-iPSC SMCs cultured in flat substrates. Our results further show that the aging effect observed in HGPS-iPSCs was negligible in N-iPSC SMCs. It is possible that N-iPSC SMCs may need more time in contact with the substrates to show significant differences in terms of aging.

Several mechanisms may account for the aging process induced by the micropatterned substrates in HGPS-iPSCs SMCs including DNA damage and oxidative stress. It is believed that the increased DNA damage is a characteristic step leading to premature aging ${ }^{34}$. Furthermore, oxidative stress may act as a trigger of DNA damage and thus promoting cell senescence ${ }^{35}$. To gain insights about the mechanism that may account for the aging process induced by the micropatterned substrates in HGPS-iPSCs SMCs we evaluated the oxidative stress caused by the micropatterned using a CellROX probe (Figure 4A). No differences were observed in terms of ROS intensity when flat and micropatterned substrate 5_5 were compared, both for HGPS-iPSCs SMCs and N-iPSCs SMCs, showing that micropatterned substrates did not induce oxidative stress to the cells. As expected, HGPS-iPSCs SMCs presented a higher ROS intensity than NiPSCs $\mathrm{SMCs}^{32}$. Furthermore, from our results we observed that the percentage of nuclei containing 5 or more H2AX foci significantly increased when HGPS-iPSCs SMCs were cultured on micropatterned substrate 5_5 compared to flat (Figure 4B and Figure S4C) but no changes 
were observed in N-iPSCs SMCs. It is interesting to note that the increase in DNA damage in HGPS-iPSCs SMCs cultured on micropatterned substrate 5_5 for 4 days was before an increase in progerin expression (Figure S6). Therefore, our results seem to indicate that the aging process observed in HGPS-iPSCs SMCs cultured in micropatterned substrates may be triggered by an increase in DNA damage. It is possible that the accumulation of SUN1 protein in HGPS-iPSC SMCs cultured on micropatterned substrate 5_5 leads to an increase activity of DNA-dependent protein kinase (DNAPK) complex which then increases the phosphorylation of histone 2A.X and DNA damage ${ }^{36}$. This leads to cellular senescence and finally to an aged cellular phenotype ${ }^{34,37}$.

In conclusion, we report a substrate with specific topographic elements that induce pathological aging in SMCs, primarily assessed by the expression of progerin but also by other markers associated with cell aging such as $\beta$-galactosidase, annexin 3 and 5 and caspase 9 (Figure 4C). We show that HGPS-iPSC SMCs cultured on micropatterned substrates (specifically 5_5) have higher expression of progerin as compared to cells cultured in flat substrates. To the best of our knowledge this is the first in vitro platform to accelerate cellular aging based on substrate topography. The current work also suggests that HGPS-iPSC SMCs cultured on microppaterned substrates have higher disruption between the cyto- and nucleoskeleton and increased DNA damage as cells cultured in flat substrates, which leads to an increase in progerin accumulation. Further experiments are required to clarify why the geometry 5_5 leads to higher cell aging than the other geometries. Although not demonstrated, it is possible that this geometry leads to a higher tension at the LINC complex. 
A

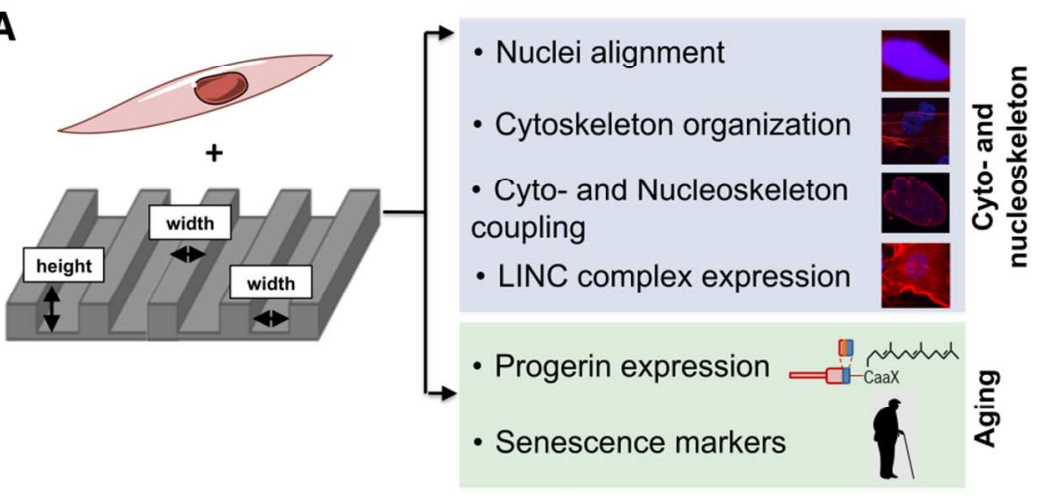

B
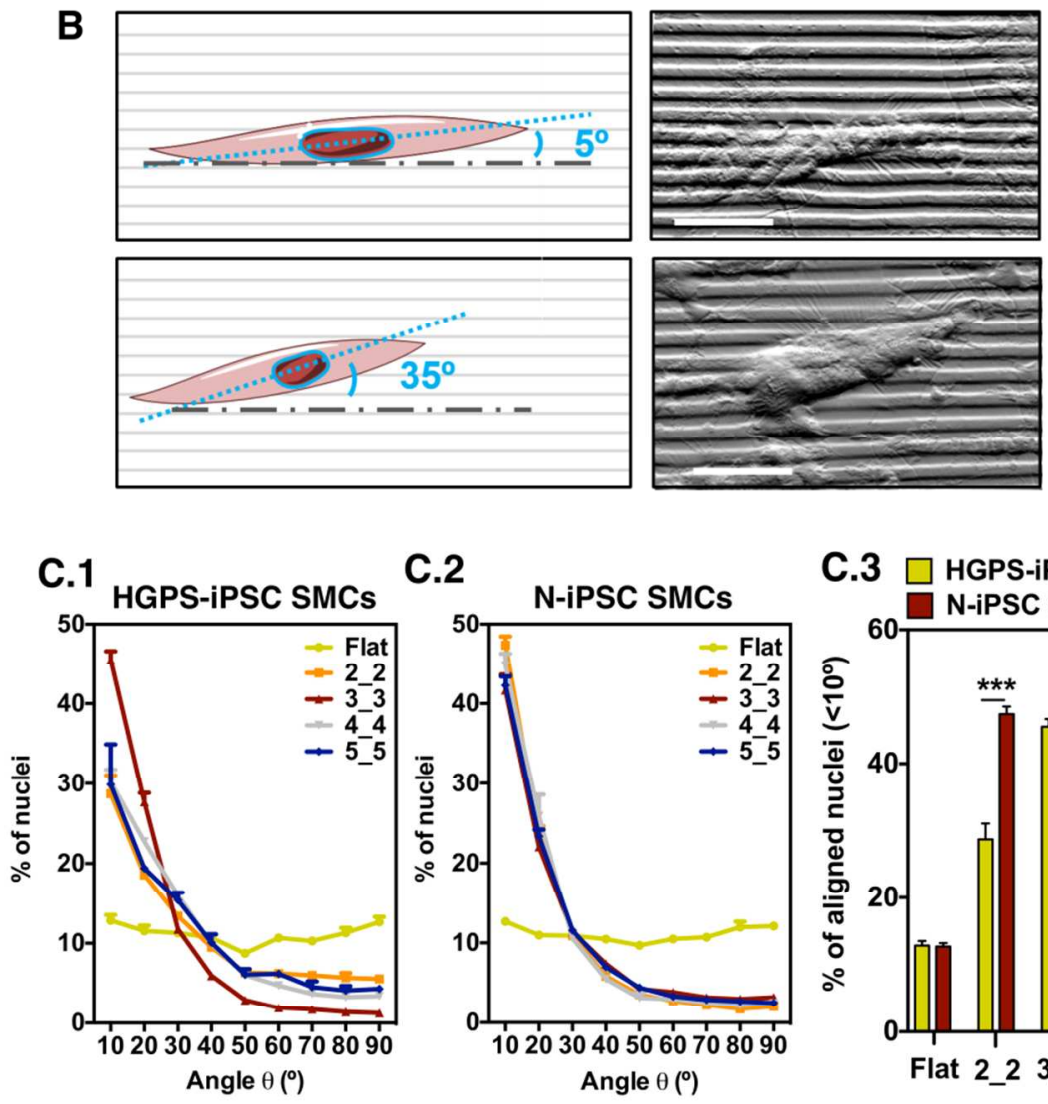

C.2
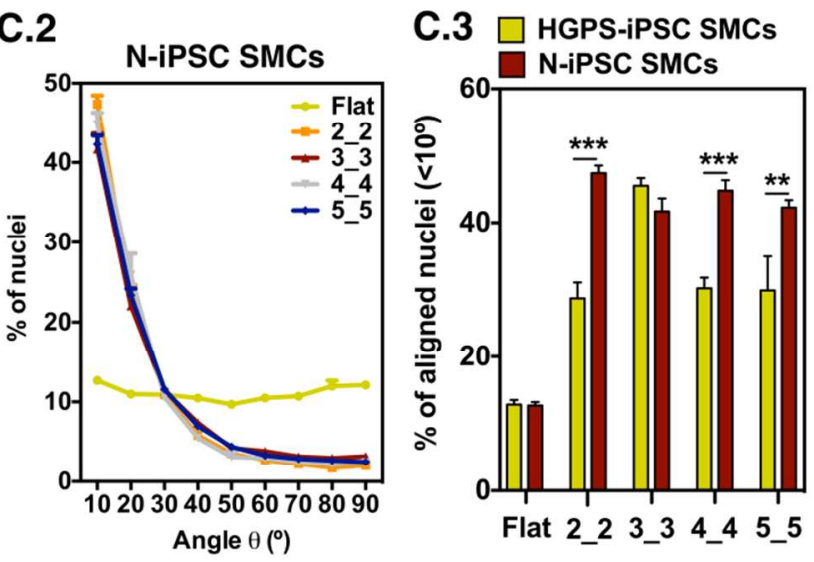

Figure 1. Effect of substrate micropatterning in cell alignment. (A) Schematic representation of the analyses performed. (B) SEM images of HGPS-iPSC-SMCs cultured for $24 \mathrm{~h}$ on micropatterned substrates. Cell alignment to the micropatterned substrates was assessed by the angle between the micropatterned substrate and the major axis of the nuclei. (C.1 and C.2) Cell nuclei alignment angles on the non-patterned and patterned structures. HGPS-iPSC-SMCs and 
$\mathrm{N}$-iPSCs SMCs were cultured for 15 days on micropatterned substrates. At least 3000 cell nuclei were counted per substrate. Three substrates have been used per condition. (C.3) Percentage of cells that have orientation angles within 0 and 10 degrees relative to the direction of the micropatterned substrate. In C.1-C.3, results are Mean \pm SEM (n=3). **, *** denotes statistical significance $(\mathrm{P}<0.01, \mathrm{P}<0.001)$ between HGPS-iPSC SMCs and N-iPSC SMCs for the same micropatterned substrate. 
A

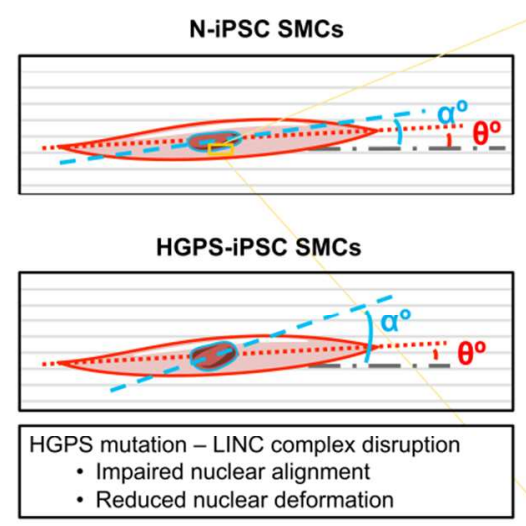

B

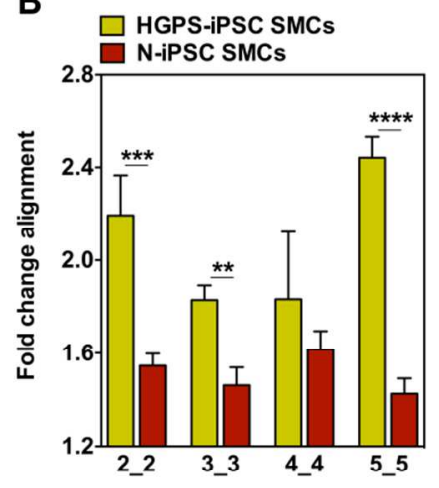

D.1 HGPS-iPSC SMCs

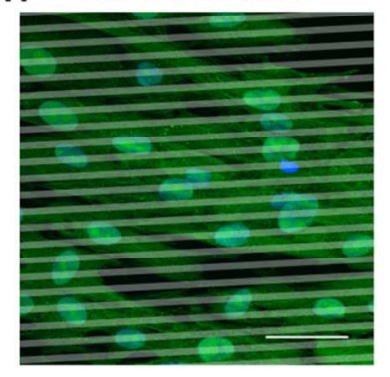

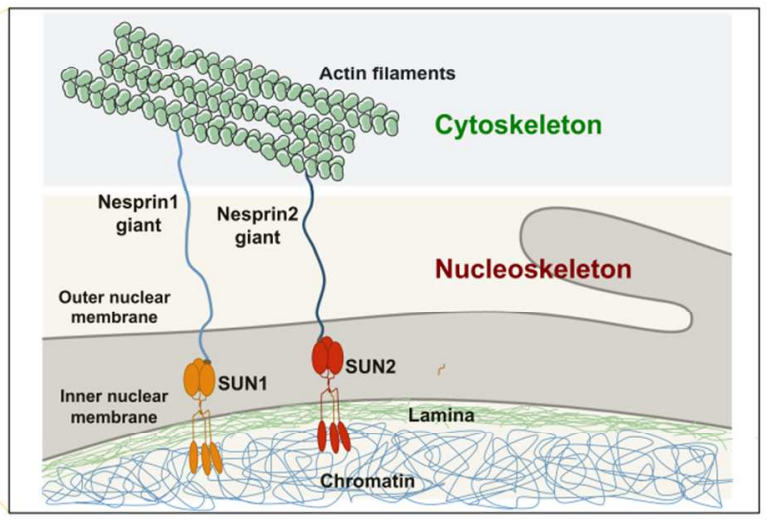

C

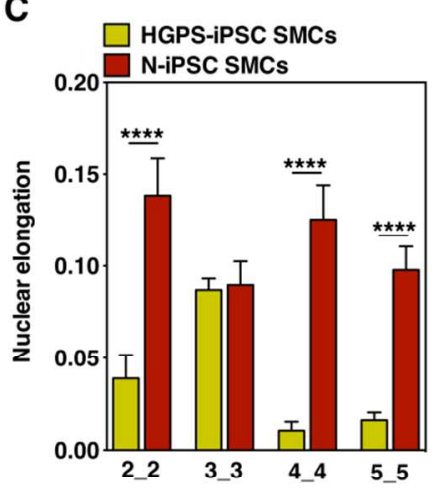

D.2 N-iPSC SMCs

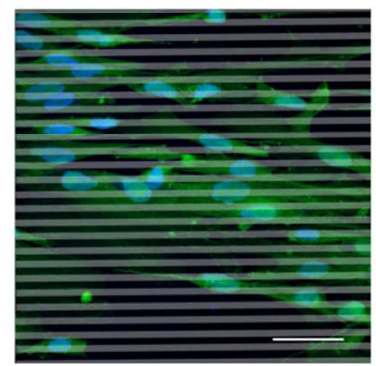

Figure 2. Effect of substrate micropatterning in cyto- and nucleoskeleton. (A) Schematic representation of the micropattern effect in the alignment of nuclei and cellular cytoskeleton. HGPS-iPSC SMCs and N-iPSC SCMs were cultured on flat and micropatterned substrates for 15 days. Cytoskeleton and nuclei alignment angles to the patterned substrates were measured. (B) The fold change alignment was calculated according to the equation: number of cells with aligned cytoplasm with respect to the micropattern $\left(\theta<10^{\circ}\right) /$ number of cells with aligned nuclei 
with respect to the micropattern $\left(\alpha<10^{\circ}\right)$. At least 300 cells were counted per substrate. Three substrates have been used per condition. (C) The elongation of the nucleus was determined by the ratio of the short axis of the nucleus to the long axis of the nucleus, for each substrate and normalized by the nonpatterned substrate. Nuclear elongation was calculated as 1-(elongation substrate/elongation nonpatterned substrate). In B and C, results are Mean $\pm \operatorname{SEM}(\mathrm{n}=3) . * *, * * *$, $* * * *$ denotes statistical significance $(\mathrm{P}<0.01, \mathrm{P}<0.001, \mathrm{P}<0.0001)$ between HGPS-iPSC SMCs and N-iPSC SMCs for the same micropatterned substrate. (D) Representative images of HGPSiPSC SMCs (D.1) and N-iPSC SCMs (D.2) alignment and nuclear elongation for 5_5 micropattern. Scale bar represents $50 \mu \mathrm{m}$. 
HGPS- IPSC SMCs

Flat 5_5
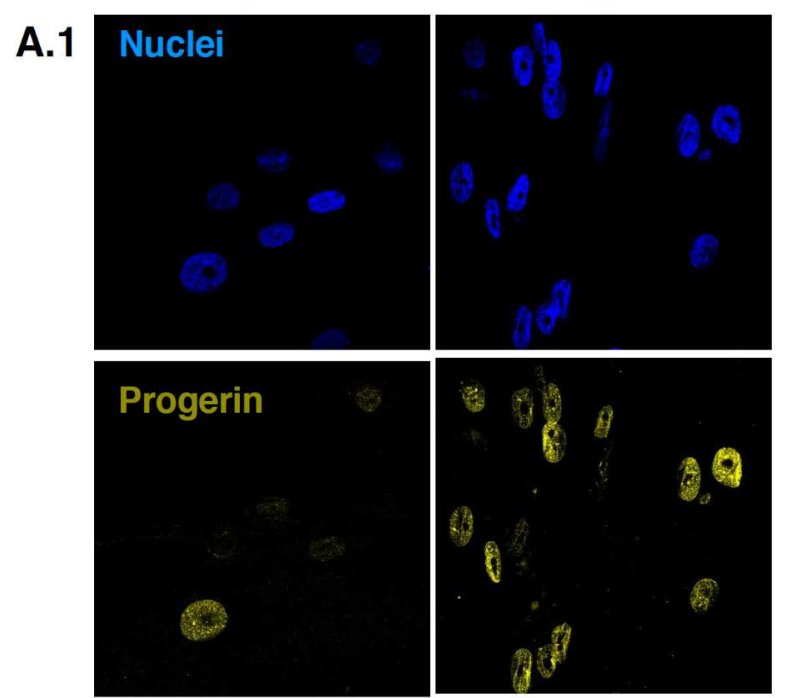

Nuclei Progerin

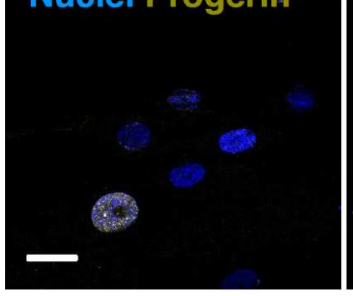

A.2
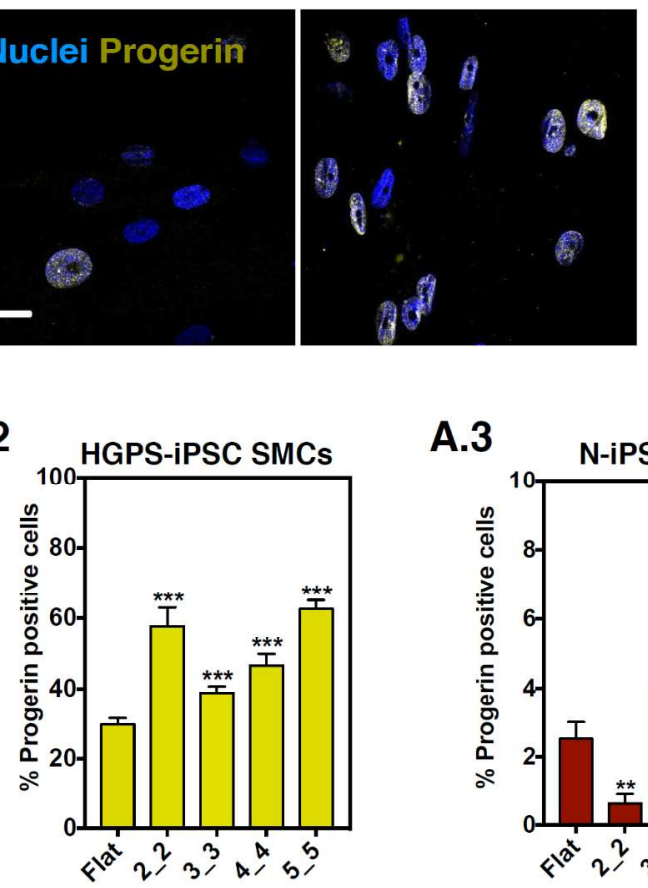

A.3

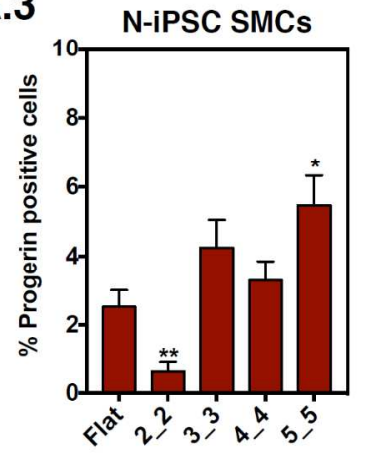

\section{$\mathrm{N}$-iPSC SMCs}

Flat
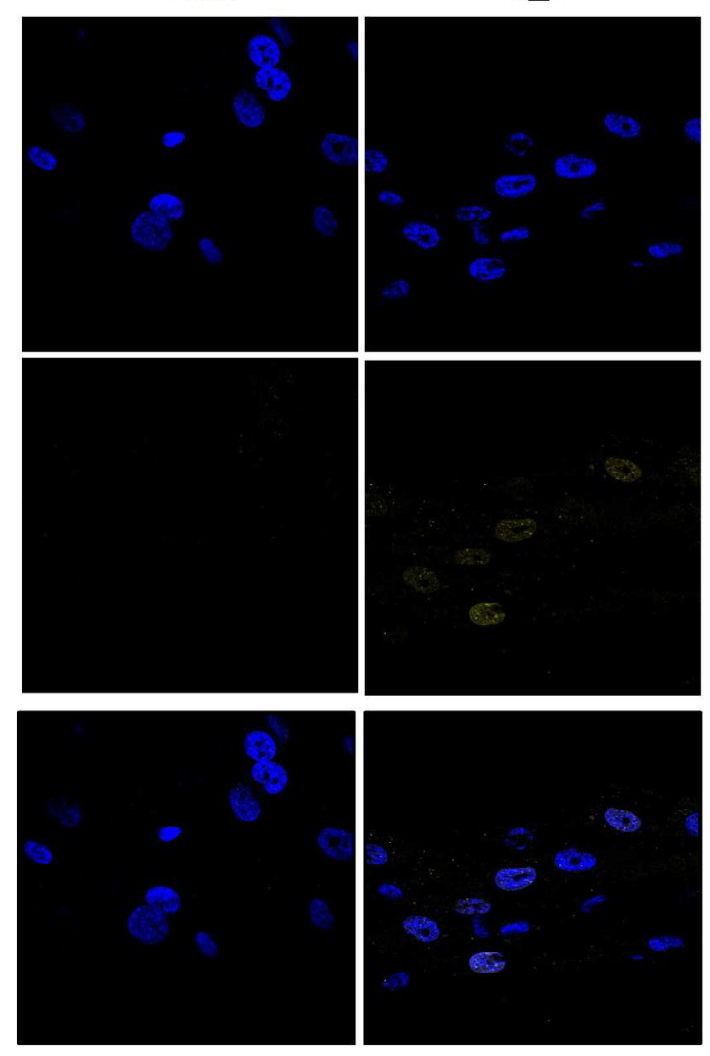

B

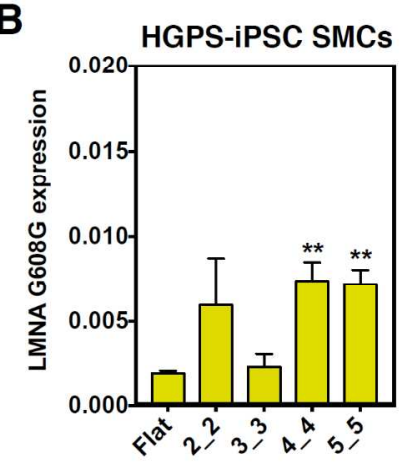

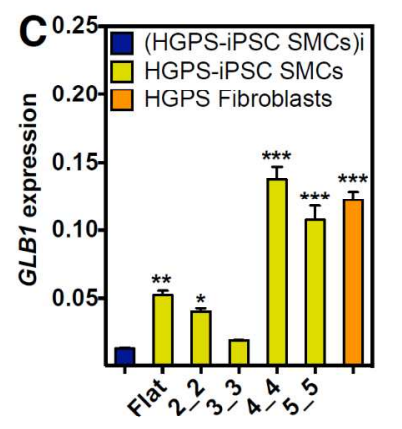
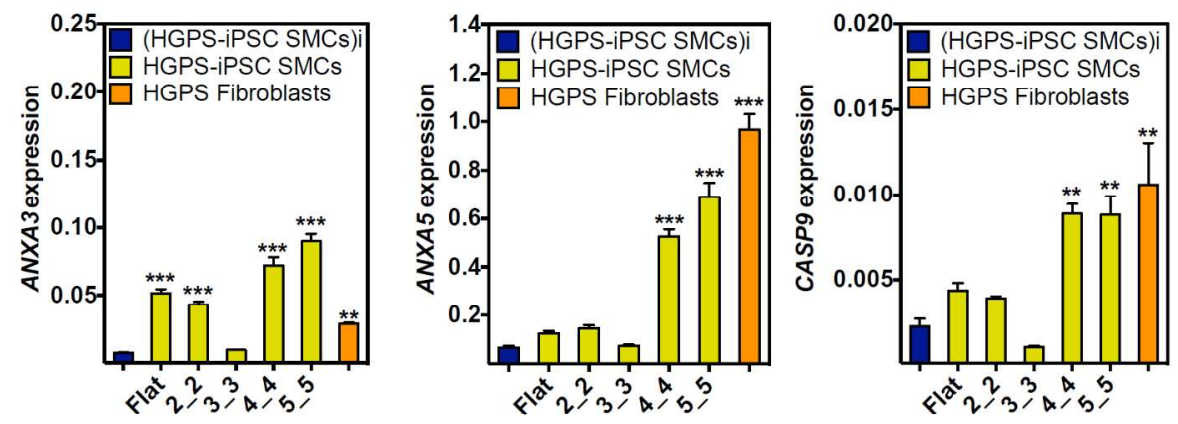

ACS Paragon Plus Environment 
Figure 3. Effect of substrate micropatterning on cell aging. (A.1) Expression of progerin by HGPS-iPSC SMCs and N-iPSC SMCs cultured for 15 days on flat and 5_5 micropatterned substrates. Scale bar is $10 \mu \mathrm{m}$. (A.2 and A.3) Percentage of cells expressing progerin as quantified by immunofluorescence. At least 3000 cell nuclei were counted per substrate. Three substrates have been used per condition. Results are Mean $\pm \operatorname{SEM}(n=3)$. (B) Expression of progerin in HGPS-iPSC SMCs by qRT-PCR. (C) Expression of aging markers GLB1, ANXA3, $A N X A 5$ and CASP9 in HGPS-iPSC SMCs as evaluated by qRT-PCR. In B and C, results are Mean \pm SEM $(n=4)$ and gene expression was normalized by the housekeeping gene GAPDH. ***,*** denotes statistical significance $(\mathrm{P}<0.05, \mathrm{P}<0.01, \mathrm{P}<0.001)$. The statistical analyses were performed between cells cultured in the substrates and initial SMCs, i.e., (HGPS-iPSC SMCs)i. 
A

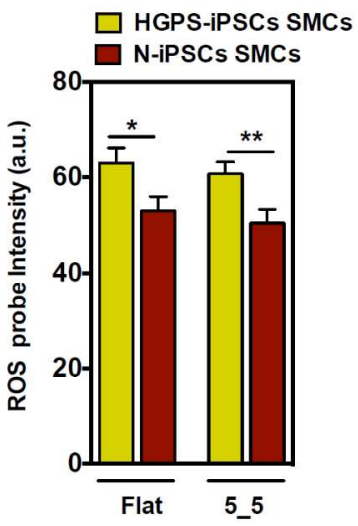

\section{B}

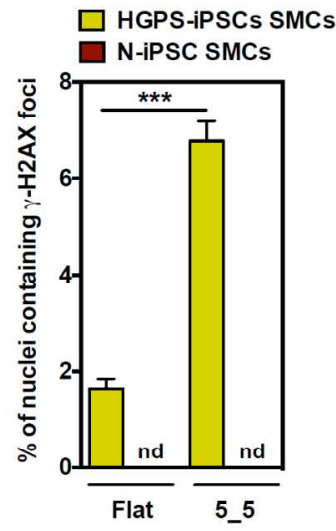

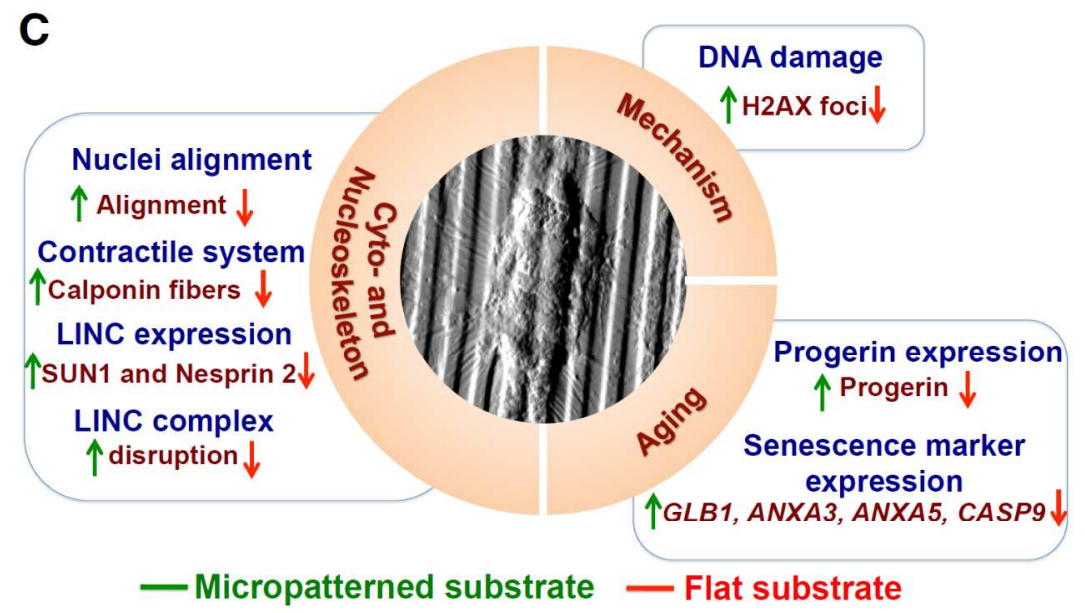

Figure 4. Effect of substrate micropatterning on ROS production and DNA damage. (A) CellROX Deep Red probe was used to quantify the oxidative stress in N-iPSCs SMCs and HGPS-iPSC SMCs cultured for $18 \mathrm{~h}$ on flat and micropatterned substrates (5_5). (B) DNA damage of HGPS-iPSCs SMCs and N-iPSCs SMCs was quantified by the percentage of nuclei containing 5 or more $\mathrm{H} 2 \mathrm{AX}$ foci after 4 days on flat and micropatterned substrates (5_5). In A and $B$ results are Mean $\pm \operatorname{SEM}(n=3)$. At least 1500 cell nuclei were counted per substrate. Three substrates have been used per condition. ${ }^{* * *}, * * *$ denotes statistical significance $(\mathrm{P}<0.05$, $\mathrm{P}<0.01, \mathrm{P}<0.001)$. (C) Summary of the results. 


\title{
Supporting Information.
}

The following files are available free of charge.

Experimental Section (PDF)

Supplementary Figures (PDF)

\section{Corresponding Author}

*Lino Ferreira.

Institute for Biomedical Imaging and Life Sciences (IBILI), Faculty of Medicine, University of Coimbra, Coimbra, Portugal. lino@uc-biotech.pt

\section{Author Contributions}

All authors have given approval to the final version of the manuscript. $\$$ These authors contributed equally.

\section{Funding Sources}

This work was supported by funds from FEDER through COMPETE program and Fundação para a Ciência e a Tecnologia (FCT) (MITP-TB/ECE/0013/2013 to L.F.; SFRH/BD/71042/2010 to P.R.P.) as well as the European project ERAatUC (ref. 669088).

\author{
ABBREVIATIONS \\ HGPS, Hutchinson-Gilford Progeria Syndrome; SMCs, smooth muscle cells; iPSCs, induced \\ pluripotent stem cells; PDMS, Poly(dimethylsiloxane); $\alpha$-SMA, $\alpha$-smooth muscle actin; \\ SMMHC, smooth muscle myosin heavy chain; DNA, deoxyribonucleic acid.
}




\section{REFERENCES}

1. Unadkat, H. V.; Hulsman, M.; Cornelissen, K.; Papenburg, B. J.; Truckenmuller, R. K.; Carpenter, A. E.; Wessling, M.; Post, G. F.; Uetz, M.; Reinders, M. J. T.; Stamatialis, D.; van Blitterswijk, C. A.; de Boer, J., An algorithm-based topographical biomaterials library to instruct cell fate. Proceedings of the National Academy of Sciences 2011, 108 (40), 16565-16570.

2. Saez, A.; Ghibaudo, M.; Buguin, A.; Silberzan, P.; Ladoux, B., Rigidity-driven growth and migration of epithelial cells on microstructured anisotropic substrates. Proceedings of the National Academy of Sciences 2007, 104 (20), 8281-8286.

3. Swift, J.; Ivanovska, I. L.; Buxboim, A.; Harada, T.; Dingal, P. C. D. P.; Pinter, J.; Pajerowski, J. D.; Spinler, K. R.; Shin, J. W.; Tewari, M.; Rehfeldt, F.; Speicher, D. W.; Discher, D. E., Nuclear Lamin-A Scales with Tissue Stiffness and Enhances Matrix-Directed Differentiation. Science 2013, 341 (6149), 1240104-1240104.

4. López-Otín, C.; Blasco, M. A.; Partridge, L.; Serrano, M.; Kroemer, G., The Hallmarks of Aging. Cell 2013, 153 (6), 1194-1217.

5. Nissan, X.; Blondel, S.; Navarro, C.; Maury, Y.; Denis, C.; Girard, M.; Martinat, C.; De Sandre-Giovannoli, A.; Levy, N.; Peschanski, M., Unique Preservation of Neural Cells in Hutchinson- Gilford Progeria Syndrome Is Due to the Expression of the Neural-Specific miR-9 MicroRNA. Cell Reports 2012, 2 (1), 1-9.

6. $\quad$ Pitrez, P. R.; Rosa, S. C.; Praça, C.; Ferreira, L., Vascular disease modeling using induced pluripotent stem cells: Focus in Hutchinson-Gilford Progeria Syndrome. Biochemical and Biophysical Research Communications 2016, 473 (3), 710-718.

7. De Sandre-Giovannoli, A.; Bernard, R.; Cau, P.; Navarro, C.; Amiel, J.; Boccaccio, I.; Lyonnet, S.; Stewart, C. L.; Munnich, A.; Le Merrer, M.; Levy, N., Lamin a truncation in Hutchinson-Gilford progeria. Science 2003, 300 (5628), 2055.

8. Scaffidi, P.; Misteli, T., Lamin A-dependent nuclear defects in human aging. Science 2006, 312 (5776), 1059-1063.

9. $\quad$ Ragnauth, C. D.; Warren, D. T.; Liu, Y.; McNair, R.; Tajsic, T.; Figg, N.; Shroff, R.; Skepper, J.; Shanahan, C. M., Prelamin A acts to accelerate smooth muscle cell senescence and is a novel biomarker of human vascular aging. Circulation 2010, 121 (20), 2200-10.

10. Rowe, V. L.; Stevens, S. L.; Reddick, T. T.; Freeman, M. B.; Donnell, R.; Carroll, R. C.; Goldman, M. H., Vascular smooth muscle cell apoptosis in aneurysmal, occlusive, and normal human aortas. J Vasc Surg 2000, 31 (3), 567-76.

11. Olive, M.; Harten, I.; Mitchell, R.; Beers, J. K.; Djabali, K.; Cao, K.; Erdos, M. R.; Blair, C.; Funke, B.; Smoot, L.; Gerhard-Herman, M.; Machan, J. T.; Kutys, R.; Virmani, R.; Collins, F. S.; Wight, T. N.; Nabel, E. G.; Gordon, L. B., Cardiovascular pathology in HutchinsonGilford progeria: correlation with the vascular pathology of aging. Arteriosclerosis, thrombosis, and vascular biology 2010, 30 (11), 2301-9.

12. Ferreira, L. S.; Gerecht, S.; Shieh, H. F.; Watson, N.; Rupnick, M. A.; Dallabrida, S. M.; Vunjak-Novakovic, G.; Langer, R., Vascular progenitor cells isolated from human embryonic stem cells give rise to endothelial and smooth muscle like cells and form vascular networks in vivo. Circ Res 2007, 101 (3), 286-94.

13. Vazao, H.; das Neves, R. P.; Graos, M.; Ferreira, L., Towards the maturation and characterization of smooth muscle cells derived from human embryonic stem cells. PLoS One 2011, 6 (3), e17771. 
14. Ribas, J.; Zhang, Y. S.; Pitrez, P. R.; Leijten, J.; Miscuglio, M.; Rouwkema, J.; Dokmeci, M. R.; Nissan, X.; Ferreira, L.; Khademhosseini, A., Biomechanical Strain Exacerbates Inflammation on a Progeria-on-a-Chip Model. Small 2017, 13 (15).

15. Marx, S. O.; Totary-Jain, H.; Marks, A. R., Vascular smooth muscle cell proliferation in restenosis. Circ Cardiovasc Interv 2011, 4 (1), 104-11.

16. Cao, Y.; Poon, Y. F.; Feng, J.; Rayatpisheh, S.; Chan, V.; Chan-Park, M. B., Regulating orientation and phenotype of primary vascular smooth muscle cells by biodegradable films patterned with arrays of microchannels and discontinuous microwalls. Biomaterials 2010, 31 (24), 6228-38.

17. Williams, C.; Brown, X. Q.; Bartolak-Suki, E.; Ma, H.; Chilkoti, A.; Wong, J. Y., The use of micropatterning to control smooth muscle myosin heavy chain expression and limit the response to transforming growth factor $\beta 1$ in vascular smooth muscle cells. Biomaterials 2011, $32(2), 410-418$.

18. Biela, S. A.; Su, Y.; Spatz, J. P.; Kemkemer, R., Different sensitivity of human endothelial cells, smooth muscle cells and fibroblasts to topography in the nano-micro range. Acta biomaterialia 2009, 5 (7), 2460-6.

19. Palmaz, J. C., Stent Technology: Understanding Molecular Characteristics is Critical. Medscape 2001.

20. Jeon, H.; Hidai, H.; Hwang, D. J.; Grigoropoulos, C. P., Fabrication of arbitrary polymer patterns for cell study by two-photon polymerization process. J Biomed Mater Res A 2010, 93 (1), 56-66.

21. Isenberg, B. C.; Backman, D. E.; Kinahan, M. E.; Jesudason, R.; Suki, B.; Stone, P. J.; Davis, E. C.; Wong, J. Y., Micropatterned cell sheets with defined cell and extracellular matrix orientation exhibit anisotropic mechanical properties. Journal of Biomechanics 2012, 45 (5), 756-761.

22. Ye, G. J. C.; Aratyn-Schaus, Y.; Nesmith, A. P.; Pasqualini, F. S.; Alford, P. W.; Parker, $\mathrm{K} . \mathrm{K}$., The contractile strength of vascular smooth muscle myocytes is shape dependent. Integrative Biology 2014, 6 (2), 152.

23. Booth, E. A.; Spagnol, S. T.; Alcoser, T. A.; Dahl, K. N., Nuclear stiffening and chromatin softening with progerin expression leads to an attenuated nuclear response to force. Soft Matter 2015, 11 (32), 6412-8.

24. Isermann, P.; Lammerding, J., Nuclear Mechanics and Mechanotransduction in Health and Disease. Current Biology 2013, 23 (24), R1113-R1121.

25. Lombardi, M. L.; Lammerding, J., Keeping the LINC: the importance of nucleocytoskeletal coupling in intracellular force transmission and cellular function. Biochemical Society Transactions 2011, 39 (6), 1729-1734.

26. Lombardi, M. L.; Jaalouk, D. E.; Shanahan, C. M.; Burke, B.; Roux, K. J.; Lammerding, J., The Interaction between Nesprins and Sun Proteins at the Nuclear Envelope Is Critical for Force Transmission between the Nucleus and Cytoskeleton. Journal of Biological Chemistry 2011, 286 (30), 26743-26753.

27. Arsenovic, P. T.; Ramachandran, I.; Bathula, K.; Zhu, R.; Narang, J. D.; Noll, N. A.; Lemmon, C. A.; Gundersen, G. G.; Conway, D. E., Nesprin-2G, a Component of the Nuclear LINC Complex, Is Subject to Myosin-Dependent Tension. Biophys J 2016, 110 (1), 34-43. 28. Chen, C.-Y.; Chi, Y.-H.; Mutalif, Rafidah A.; Starost, Matthew F.; Myers, Timothy G.; Anderson, Stasia A.; Stewart, Colin L.; Jeang, K.-T., Accumulation of the Inner Nuclear 
Envelope Protein Sun1 Is Pathogenic in Progeric and Dystrophic Laminopathies. Cell 2012, 149 (3), 565-577.

29. Lei, K.; Zhu, X.; Xu, R.; Shao, C.; Xu, T.; Zhuang, Y.; Han, M., Inner Nuclear Envelope Proteins SUN1 and SUN2 Play a Prominent Role in the DNA Damage Response. Current Biology 2012, 22 (17), 1609-1615.

30. Lee, B. Y.; Han, J. A.; Im, J. S.; Morrone, A.; Johung, K.; Goodwin, E. C.; Kleijer, W. J.; DiMaio, D.; Hwang, E. S., Senescence-associated $\beta$-galactosidase is lysosomal $\beta$-galactosidase. Aging Cell 2006, 5 (2), 187-195.

31. Klement, K.; Melle, C.; Murzik, U.; Diekmann, S.; Norgauer, J.; Hemmerich, P., Accumulation of annexin A5 at the nuclear envelope is a biomarker of cellular aging. Mechanisms of Ageing and Development 2012, 133 (7), 508-522.

32. Trigueros-Motos, L., Hutchinson-Gilford progeria syndrome, cardiovascular disease and oxidative stress. Frontiers in Bioscience 2011, S3 (1), 1285.

33. Zhang, J.-H.; Zhang, Y.; Herman, B., Caspases, apoptosis and aging. Ageing Research Reviews 2003, 2 (4), 357-366.

34. Liu, B.; Wang, J.; Chan, K. M.; Tjia, W. M.; Deng, W.; Guan, X.; Huang, J.-d.; Li, K. M.; Chau, P. Y.; Chen, D. J.; Pei, D.; Pendas, A. M.; Cadiñanos, J.; López-Otín, C.; Tse, H. F.; Hutchison, C.; Chen, J.; Cao, Y.; Cheah, K. S. E.; Tryggvason, K.; Zhou, Z., Genomic instability in laminopathy-based premature aging. Nature Medicine 2005, 11 (7), 780-785.

35. Barascu, A.; Le Chalony, C.; Pennarun, G.; Genet, D.; Zaarour, N.; Bertrand, P., Oxydative stress alters nuclear shape through lamins dysregulation. Nucleus 2012, 3 (5), 411417.

36. Lei, K.; Zhu, X.; Xu, R.; Shao, C.; Xu, T.; Zhuang, Y.; Han, M., Inner nuclear envelope proteins SUN1 and SUN2 play a prominent role in the DNA damage response. Curr Biol 2012, 22 (17), 1609-15.

37. Chen, J. H.; Hales, C. N.; Ozanne, S. E., DNA damage, cellular senescence and organismal ageing: causal or correlative? Nucleic Acids Res 2007, 35 (22), 7417-28. 


\section{FOR TABLE OF CONTENTS ONLY}

\section{SUBSTRATE TOPOGRAPHY MODULATES CELL AGING ON A PROGERIA CELL MODEL}

Patricia R. Pitrez ${ }^{1,2 \hbar}$, Luís Estronca ${ }^{1,2 \hbar}$, Helena Vazão ${ }^{1}$, Anna-Laure Egesipe ${ }^{3}$, Amélie Le Corf $^{3}$,

Claire Navarro ${ }^{4}$, Nicolas Lévy ${ }^{5}$, Annachiara De Sandre-Giovannoli ${ }^{5}$, Xavier Nissan ${ }^{3}$, Lino

Ferreira $^{1,2 *}$

${ }^{1}$ Center for Neuroscience and Cell Biology, University of Coimbra, Coimbra, Portugal

${ }^{2}$ Institute for Biomedical Imaging and Life Sciences (IBILI), Faculty of Medicine, University of Coimbra, Coimbra, Portugal

${ }^{4}$ Aix Marseille Univ, INSERM, GMGF, Marseille, France

${ }^{3}$ CECS, I-STEM, AFM, Institute for Stem Cell Therapy and Exploration of Monogenic diseases, Evry cedex, France

${ }^{5}$ Aix Marseille Univ, INSERM, GMGF, Marseille, France and Molecular Genetics Laboratory, Department of Medical Genetics, La Timone Children's hospital, Marseille, France.

\section{Micropatterned vs flat substrates}
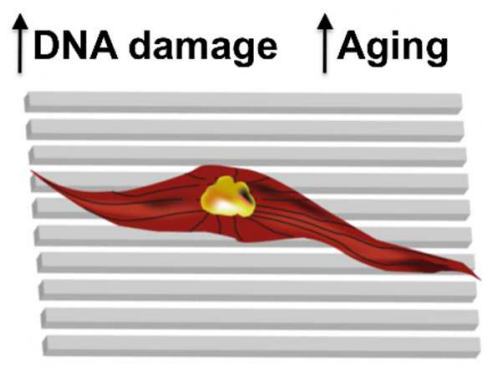